\title{
Implementation of Pulse Shaping Techniques in OFDM System
}

\author{
Divyangna Gandhi \\ M.Tech. student \\ Sardar Vallabhbhai National \\ Institute of technology, Surat
}

\author{
Shilpi Gupta \\ Assistant Professor \\ Sardar Vallabhbhai National \\ Institute of technology, Surat
}

\author{
Upena Dalal \\ Associate Professor \\ Sardar Vallabhbhai National \\ institute of technology, Surat
}

\begin{abstract}
Tremendous improvement has been made possible in wireless technology due to success of OFDM system which is a multi carrier modulation technique with capability to support high data rates. Despite its many advantages OFDM has failed against inter symbol interference (ISI), high sensitivity to Inter carrier interference (ICI), high peak to average power ratio (PAPR), phase noise and band limited channel. Hence the performance of an OFDM system is highly affected by the aforementioned undesired phenomenon. For this reason bandlimited system often employs pulse shaping techniques. In this paper pulse shaping of multi carrier signal is introduced by using various pulse shapes like Rectangular pulse (REC), Raised cosine pulse (RC), Better than raised cosine pulse (BTRC), Sinc power pulse (SP) and Improved sinc power pulse (ISP). The impulse responses and frequency spectrums have been evaluated and compared with system without using pulse shaping. Results obtained by simulation for OFDM system using pulse shaping and without using pulse shaping indicate that the performance of OFDM system with various pulse shapes is better than without using pulse shaping. Along with it can also be observed that among pulse shaping ISP exhibits better performance. Computer simulation has been done by using MATLAB 7.0 software.
\end{abstract}

\section{Keywords}

OFDM, ISI, ICI, Rectangular pulse (REC), Raised cosine pulse (RC), Better than raised cosine pulse (BTRC), Sinc power pulse (SP) and Improved sinc power pulse (ISP).

\section{INTRODUCTION}

It has been proven that high data speed transmission in mobile environment is achieved by Orthogonal Frequency Division Multiplexing (OFDM) system due to its ability to decompose a wideband frequency selective fading channel into several parallel narrow band flat fading channels $[1,2]$. Multi-path delay spread tolerance, immunity to frequency selective fading channels, high spectral efficiency (by allowing the sub carriers to overlap in the frequency domain), efficient modulation and demodulation techniques and robust to impulse noise are the leading advantages of OFDM system [3, 4]. OFDM technique has potential of enhancing the data rate in band limited channel. However, inter-carrier interferences (ICI) and high peak to average power ratio (PAPR) are main drawbacks of OFDM system [2,3].

Over the last decade a lot of research has been carried out in reducing the two major limitations of OFDM to enhance the performance of the system. One of them is the sensitivity of OFDM signal against carrier frequency offset which causes ICI $[2,3,4,5,6,7,8]$ and another one is the large variation in envelope of OFDM signal, which causes high peak-to-average power ratio (PAPR) $[2,3]$.

The performance of OFDM system can be made better by using efficient Pulse shaping techniques. Simulation results show that with various pulse shapes the performance of OFDM is improved compared to without pulse shaping functions and also it is observed that in comparison to the other pulse shapes like REC, RC, BTRC and SP, ISP pulse shape provides better improvement.

In this paper, Section 2 introduces OFDM System model. Different pulse shaping functions are listed in Section 3. Section 4 includes simulation results. Lastly, Section 5 concludes the paper.

\section{SYSTEM MODEL}

Figure 1 shows the structure of a typical OFDM communication system with various pulse shaping functions. In this system QPSK constellation is used to mapped binary information. The high speed serial data stream is split up into a set of low speed sub streams and modulated onto the orthogonal carriers through Inverse Fast Fourier transform (IFFT). Signal $\mathrm{s}(\mathrm{t})$ which is represented as in (2.1) is transmitted through the channel with various pulse shaping functions [2].

OFDM block with pulse-shaping is represented as:

$$
s(t)=e^{j 2 \pi f_{c} t} \sum_{k=0}^{N-1} D_{k} p(t) e^{j 2 \pi f_{k} t}
$$

Where $\mathrm{j}=\sqrt{-1}$, Number of subcarrier is $\mathrm{N}$, Carrier frequency of OFDM system is $f_{c}$, Subcarrier frequency of the $K_{t h}$ subcarrier is $f_{k}$, Where $k=0,1 \ldots N-1$, Time limited pulse shaping function is $\mathrm{p}(\mathrm{t})$. Transmitted symbol is $D_{k}$ which is assumed to have zero mean and normalized average symbol energy. Also we consider that all data symbols are uncorrelated $[9,10]$ i.e.:

$$
E\left[D_{k} D^{*}{ }_{m}\right]= \begin{cases}1, & k=m, k, m=0,1, \ldots ., N-1 \\ 0, & k \neq m, k, m=0,1, \ldots, N-1\end{cases}
$$

Where $D^{*}{ }_{k}$ is the complex conjugate of $D_{k}$. To ensure the orthogonality of subcarrier, it is very important to satisfied the below equation for OFDM system $[9,10]$. The subcarrier 


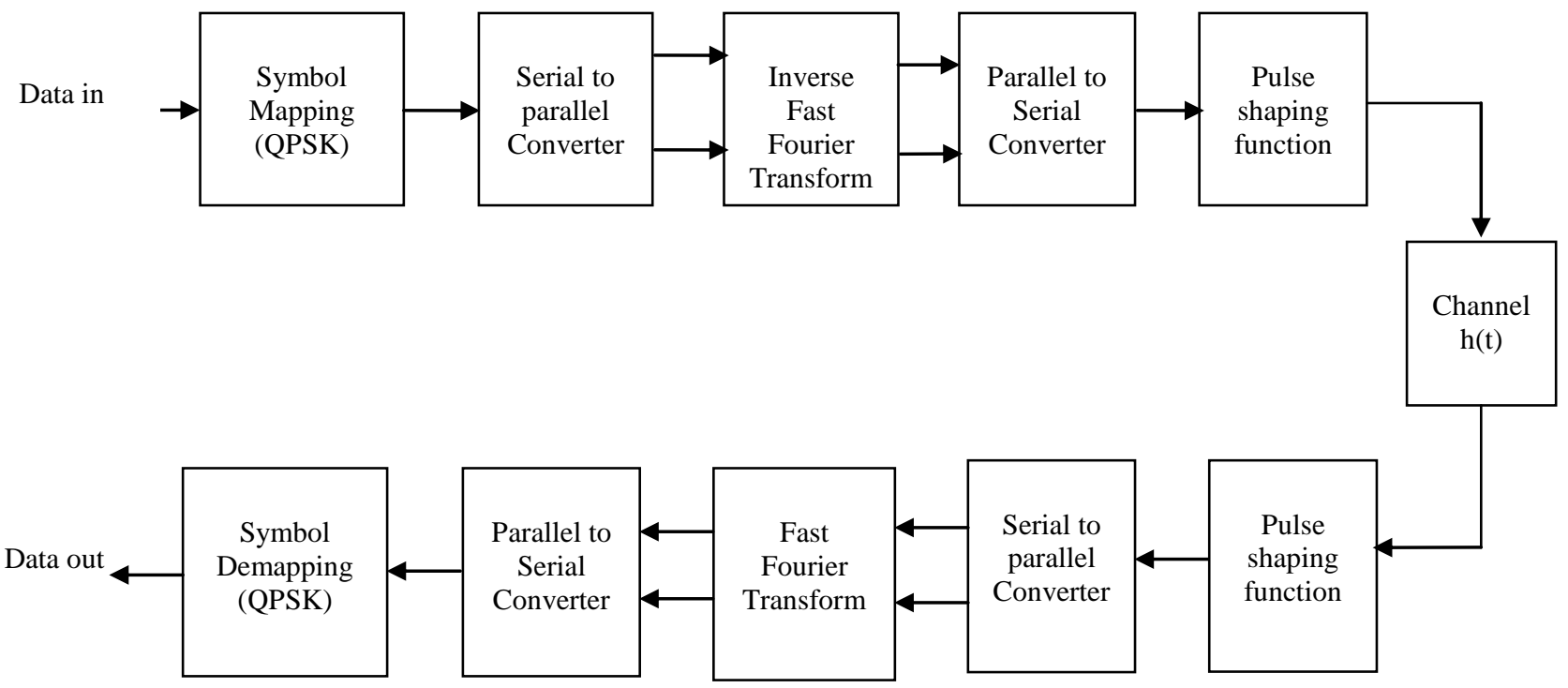

Figure 1 Simulation block diagram of OFDM System

frequency is

$$
\begin{array}{ll}
f_{k}=\frac{k}{T_{s}} ; & k=0,1, \ldots . N-1 \\
f_{k}-f_{m}=\frac{k-m}{T_{s}}, & k, m=0,1, \ldots \ldots . N-1
\end{array}
$$

For maintaining orthogonality between subcarriers, the minimum required subcarrier frequency spacing is $1 / T_{s}$. The received signal at the receiver can be represented as:

$r(t)=s(t) \otimes h(t)+w(t)$

In above equation convolution is denoted by $\otimes, \mathrm{h}(\mathrm{t})$ is the channel impulse response and the additive white Gaussian noise is represented by $w(t)$ which process with zero mean and variance $N_{0} / 2$ per dimension.

\section{PULSE SHAPING FUNCTION}

Each carrier in the OFDM spectrum is represented by main lobe with a number of side lobes having lower amplitudes. Since peak power is associated with main lobe and ICI power is associated with side lobes, so the motive of pulse shaping function is to increase the width of main lobe and/or reduce the amplitude of sidelobes [9]. Proper pulse shaping techniques makes a digital communication system possible to transmit data within a limited BW with minimum ISI [11].

In this section some most commonly used pulse shaping functions have been introduced. These functions are REC, RC, BTRC, SP and ISP. Their Fourier transforms are given, respectively as $[2,3,4,7,9]$.
The various pulse shaping functions are

$$
\begin{aligned}
& P_{R E C T}(f)=\sin c(f T) \\
& P_{S P}=\sin c^{n}(f T) \\
& P_{R c}(f)=\sin c(f T) \frac{\cos (\pi \alpha f T)}{1-(2 \alpha f T)^{2}} \\
& P_{B T R C}(f)=\sin c(f T) \frac{2 \beta f T \sin (\pi \alpha f T)+2 \cos (\pi \alpha f T)-1}{1+(\beta f T)^{2}}
\end{aligned}
$$

$P_{I S P}(f)=\exp \left(-a(f T)^{2}\right) \sin c^{n}(f T)$

Where ' $\alpha$ ' is the roll-off factor $(0 \leq \alpha \leq 1)$. To adjust the amplitude of improved sinc power pulse design parameter ' $a$ ' is used in equation (3.5), the degree of sinc power pulse is ' $n$ ' and constant parameter of BTRC pulse is denoted by ' $\beta$, $(\beta=\pi \alpha / \ln 2)[2,3,6,7,9]$.

ISP has fast decaying rate with decreasing the lobes of SP function further which is acquired with altering SP with $\exp \left(-a(f T)^{2}\right)$ as seen in (3.2) and (3.5) and thus make the average ICI power more lesser than SP[7].

\section{SIMULATION RESULTS}

The simulation results have been done to demonstrate the performance enhancement of shaping the OFDM system using the various time-limited pulse shaping functions which have been mentioned in section 3 . 


\subsection{Comparison of impulse responses.}

\subsubsection{Comparison of various pulse shapes.}

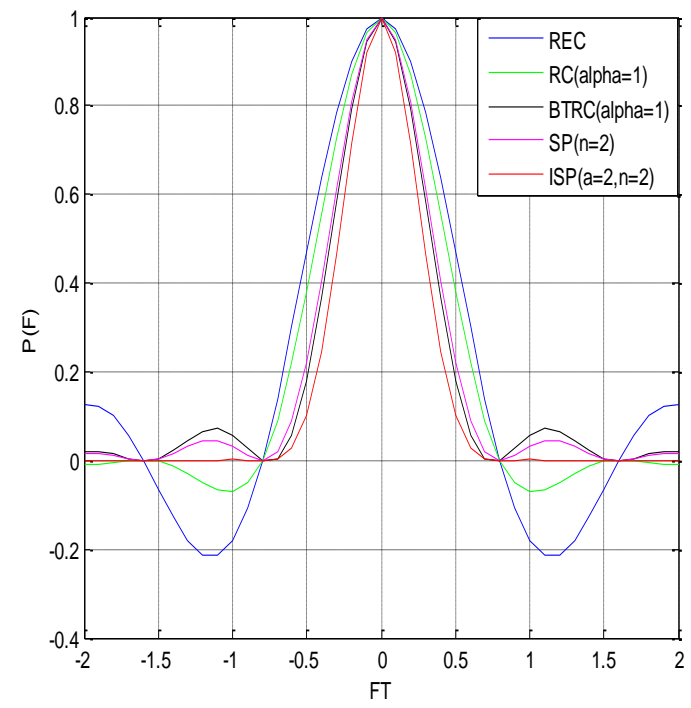

Figure 2 Comparison of Impulse response for all pulses.

Figure 2 represents comparison for impulse response of REC, RC, BTRC, SP, and ISP pulse shapes with $\alpha=1, \mathrm{a}=2$ and $\mathrm{n}=2$. It is observed that width of ISP main lobe is narrower in time domain as compared to other existing pulses which leads less ISI. It can also be observed that rectangular pulse shape has maximum and ISP pulse shape has minimum sidelobes amplitude. So ISP pulse shape is less affected by ICI power than those of other pulse shapes because lesser the side lobes lesser the ICI power. Also it has a fast decaying rate with decreasing the sidelobes of sinc function. These properties help in providing better performance due to both ICI and PAPR reduction.

\subsubsection{Comparison of ISP pulse shape for different} value of $a$.

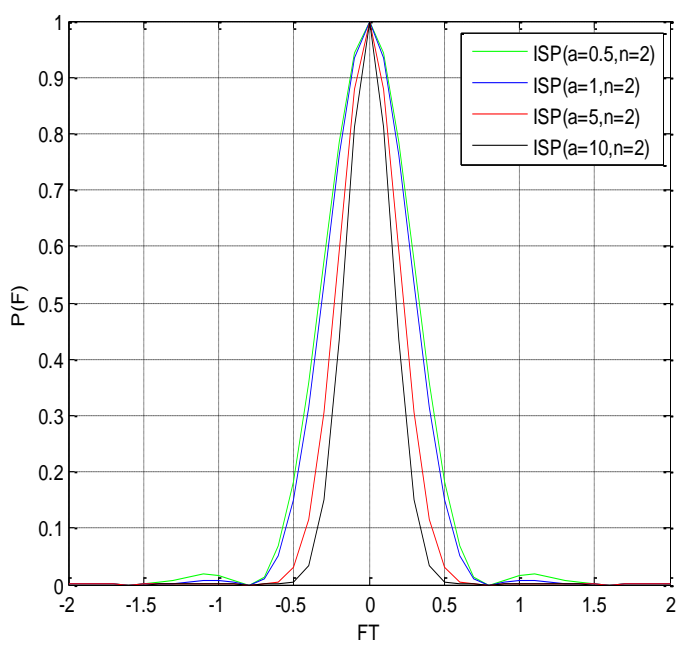

Figure 3 comparison of ISP pulse shape with different values of $\mathbf{a}$.
ISP pulse with different values of ' $a$ ' $(0.5,1,5,10)$ is depicted in Figure 3 where the amplitude of ISP pulse is adjusted by varying a design parameter ' $\mathrm{a}$ ' and $n$ is the degree of the sinc function. As the value of design parameter a increases from 0.5 to 10 , the width of the main lobe is further reducing up to some extent. But after that for very large values of a, ISP pulse shape become to a very narrow pulse shape which is not preferred for the system having frequency offset.

\subsubsection{Comparison of REC, $R C$ and BTRC with different value of $\alpha$.}

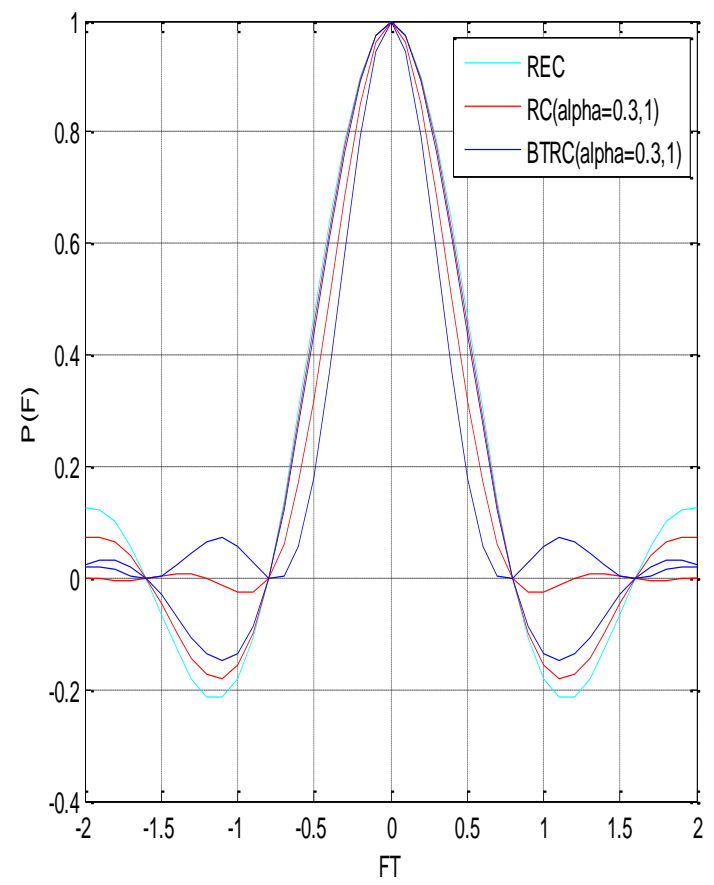

Figure 4 Comparison of REC, RC and BTRC.

To observe the effect of various values of $\alpha$, simulation has been done for $\alpha=0,0.3$ and 1. Results have been shown in Figure 4. When $\alpha=0$ the raised cosine and the BTRC pulse merge in to a rectangular pulse. When $\alpha=0.3$ the width of main lobe and amplitude of side lobe are higher than that of $\alpha=1$ for both RC and BTRC. So the width of main lobe and amplitude of sidelobes can be reduced by increasing the value of $\alpha$ from 0 to 1 . Hence the ICI power of the pulse can be diminished.

\subsection{Frequency spectrum comparison with and without pulse shaping functions.}

Simulation parameters which have been used are as follows: FFT bin size: 1024; No. of subcarriers: 210; symbols per subcarrier: 50 and 2 bits per symbol. and Channel: AWGN. The cyclic prefix added is $25 \%$ of the FFT bin size. SNR: 1 to 30.

In Figures 5 and 6 Frequency spectrum of OFDM system have been analyzed without using any pulse shaping function and using different pulse shaping functions individually. 


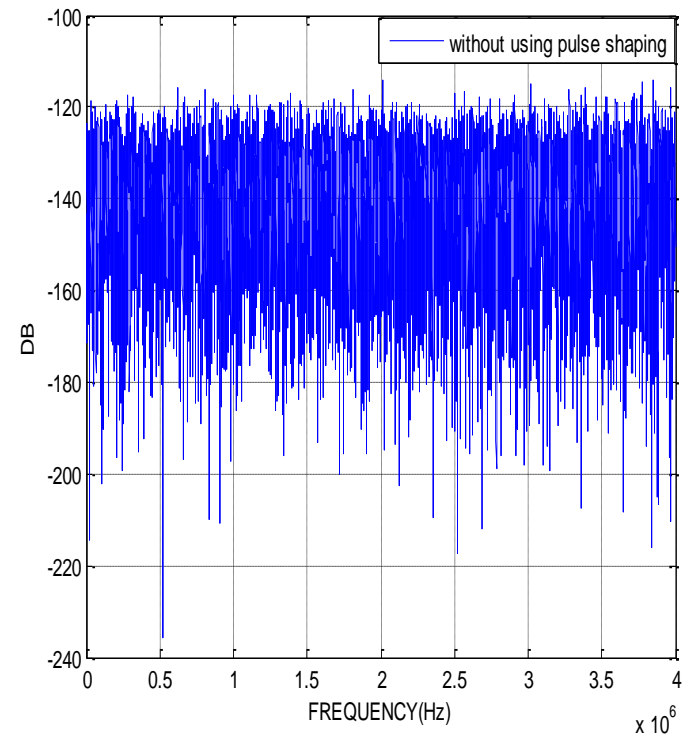

Figure 5 Frequency spectrum without using pulse shaping in OFDM system.

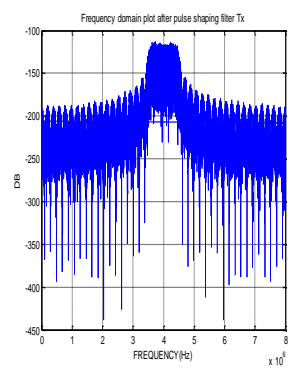

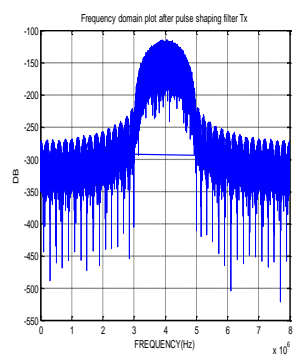
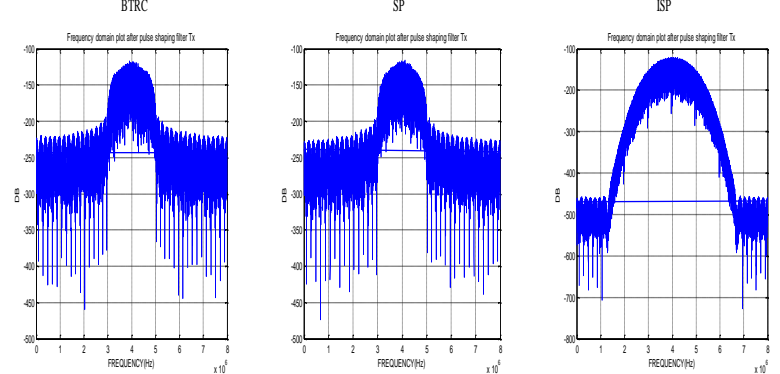

Figure 6 Frequency spectrums for individual pulse shaping functions in OFDM system.

From Figures 5 and 6 it can be observed that without pulse shaping function the spectrum is flat and the main lobe and side lobes cannot be distinguished properly. But while considering various pulse shaping functions ISP gives better spectrum because main lobe width and amplitude is higher than rest of other in frequency domain. So it is found that ISP is more spectrum efficient. Frequency spectrum using different pulse shaping functions has been shown in Figure 7.

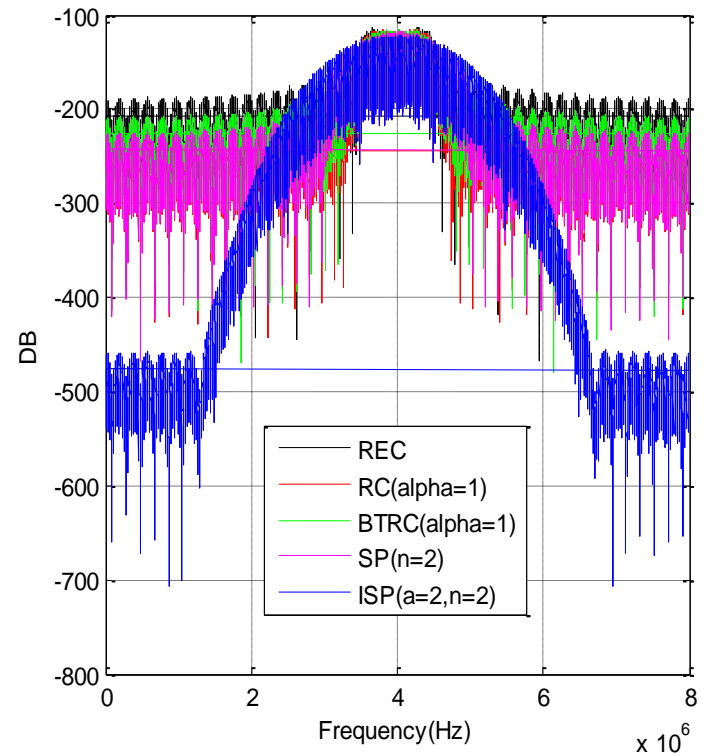

Figure 7 Combined Frequency spectrum of different pulse shaping functions.

\section{CONCLUSION}

In this work, simulation results have been presented to demonstrate that shaping the OFDM pulse significantly enhances the performance of OFDM system as compared to without using pulse shaping. It can be concluded that if pulse shaping function has the spectrum with higher width of main lobe and smaller amplitude of side lobes than others in frequency domain, then that pulse shaping function will lead less ICI and better bandwidth efficiency.

From the Simulation results it can be observed that ISP pulse has both the above mentioned features so it can be a better option in comparison to other pulse shapes.

\section{REFERENCES}

[1] S. D. Assimonis, M. Matthaiou, G. K. Karagiannidis and J. A. Nossek, "Optimized Better Than Raised-Cosine Pulse for Reduced ICI in OFDM Systems", in 17th International Conference on Telecommunications, 2010.

[2] M.Palaivelan, S. Anand and S. P. Venkatesan. 2012. "PAPR and ICI Reduction in OFDM Systems using Modified Raised Cosine Power Pulse Shape", European Journal of Scientific Research ISSN 1450-216X Vol.72 No.4 (2012), pp. 618-627.

[3] Srabani Mohapatra and Susmita Das. 2009. "A New Approach For Performance Improvement of OFDM System Using Pulse Shaping", M.Tech Thesis.NIT Rourkela.

[4] S. Aenagandula, A. Kumar, Srinivas K. and M. Nanda, "Inter Carrier Interference and Signal to Interference Ratio of various Pulse Shaping Functions used in OFDM System with Carrier Frequency Offset", International Journal of Electronics Signals and Systems (IJESS) ISSN: 2231-5969, Vol-1 Iss-3, 2012.

[5] J. Armstrong. "Analysis of new and existing methods of reducing intercarrier interference due to carrier frequency offset in OFDM", IEEE Transactions on 
Communications, vol. 47, no. 3, pp. 365-369, Mar1999.

[6] H.M. Mourad. 2006. "Reducing ICI in OFDM systems using a proposed pulse shape", Wireless Person. Communications. (2006)40: 41-48.

[7] Volkan Kumbasar and O־guz Kucur. 2007. "ICI reduction in OFDM systems by using improved sinc power pulse", Digital Signal Processing 17 (2007) 9971006.

[8] Srabani Mohapatra and Susmita Das, "Performance Enhancement of OFDM System with ICI Reduction Technique", In Proceedings of the World Congress on Engineering, Vol. I, July, 2009.
[9] Nadieh M. Moghaddam and Mohammad Mohebbi, "ICI Reduction Methods in OFDM Systems," Recent Advance in wireless Communication and Networks, Prof. Jia-Chin Lin (Ed.), ISBN: 978-953-307-274-6, In Tech.

[10] P. Tan, N.C. Beaulieu. 2004. "Reduced ICI in OFDM systems using the better than raised-cosine pulse", IEEE Commun. Lett. 8 (3) (2004) 135-137.

[11] Mahua Pal, "Algorithm for sharpening Raised Cosine Pulse shaping Digital Filter and Analysis of Performance Of QAM system when subjected to Sharpened Raised Cosine Filter", International Journal Of Scientific and Research Publication, Volume 2, Issue 1, January 2012. ISSN 2250-3153. 